REGARDS

SUR LECONOMIE ALLEMANDE

BULLETIN ECONOMIQUE DU CIRAC
Regards sur l'économie allemande

Bulletin économique du CIRAC

72 | 2005

Varia

\title{
Le changement nécessaire
}

\section{Isabelle Bourgeois}

\section{OpenEdition}

Journals

Édition électronique

URL : http://journals.openedition.org/rea/242

DOI : $10.4000 /$ rea. 242

ISBN : 978-2-8218-0840-9

ISSN : 1965-0787

Éditeur

CIRAC

Édition imprimée

Date de publication : 1 juillet 2005

Pagination : 3-6

ISSN : 1156-8992

Référence électronique

Isabelle Bourgeois, « Le changement nécessaire », Regards sur l'économie allemande [En ligne], 72 | juillet 2005, mis en ligne le 19 juin 2008, consulté le 15 septembre 2020. URL : http:// journals.openedition.org/rea/242 


\section{Le changement nécessaire}

Difficile de dire, en ce début d'été, comment va évoluer précisément sur l'année la conjoncture allemande. Alors que, dans leur rapport de printemps conjoint publié fin avril, les six Instituts tablaient encore sur une croissance de $+0,7 \%$ pour 2005, ils se montrent plus réservés deux mois après - tout en maintenant cependant leurs prévisions chiffrées. Diverses raisons les incitent à la prudence: d'une part, l'évolution de l'économie européenne dans son contexte mondial; d'autre part, la nouvelle donne intérieure créée par l'échec électoral de la coalition SPD/Verts le 22 mai dans le Land de Rhénanie du Nord-Westphalie et l'annonce consécutive d'élections anticipées au Bundestag courant septembre.

Les incertitudes portent sur le second semestre 2005. Car au cours du premier trimestre, le PIB allemand avait enregistré une croissance de $+1 \%$ (données CVS) ; en glissement annuel, le PIB a même crû de $+4,2 \%$. C'est une forte embellie après la stagnation de la fin 2004, et qui renoue avec les performances du début 2001. Mais elle pourrait être de courte durée, étant donné que la croissance mondiale a perdu en vigueur, contribuant au ralentissement conjoncturel que connaît la zone euro, c'est-à-dire le principal client de l'Allemagne, avec le risque d'un tassement des exportations allemandes.

Ces dernières restent néanmoins solides : en mai 2005 (derniers chiffres publiés par Destatis), elles ont été en hausse $+5,3 \%$ par rapport à mai 2004 , et de $+3,8 \%$ par rapport à avril 2005. II est vrai que ce mois-là, elles avaient légèrement baissé $(-0,5 \%)$. Mais comme le commerce extérieur a fluctué fortement au cours des derniers mois, il faut attendre les statistiques du premier semestre pour avoir une meilleure image de leur évolution. Quoi qu'il en soit, depuis le milieu de l'an passé (année record, il faut le rappeler), on constate un léger ralentissement des exportations allemandes, explique le ministère fédéral de l'Economie. Et de fait, les entrées en commandes dans l'industrie, qui subissent elles aussi un mouvement en dents de scie, poursuivent le léger recul amorcé voici quelque temps déjà : selon Destatis, elles ont baissé de $-0,2 \%$ entre avril/ mai et février/mars 2005, alors que si on considère la variation mai/avril seulement, elles ont été en hausse de $+2,7 \%$, tirées par les commandes extérieures $(+5,0 \%)$. Cette situation incite les entreprises à garder dans l'ensemble leur optimisme.

La production industrielle a enregistré $+0,9 \%$ en comparaison bimestrielle, une hausse à mettre principalement à l'actif des biens intermédiaires $(+1,8 \%)$, un secteur-clef des exportations allemandes. Dans l'ensemble, malgré une demande intérieure toujours aussi faible, la production industrielle, tributaire essentiellement de la demande extérieure, reste donc orientée à la hausse en tendance générale, avec $+2,3 \%$ en avril/mai 2005 par rapport aux mêmes mois de 2004. Le BTP, pour sa part, continue de reculer $(-6,7 \%$ par rapport à la même période), bien que les activités aient repris avec le retour du printemps (+10,2 \% d'avril/mai à janvier/février 2005).

Dans ce contexte somme toute favorable, à quoi il faut ajouter des taux d'intérêt faibles, une modération salariale qui se poursuit depuis une décennie, de même qu'une légère baisse des prélèvements fiscaux et sociaux à la suite des réformes, les entreprises se remettent à investir pour étendre leurs capacités. Bien que réelle, cette tendance est encore ténue, les entreprises étant actuellement préoccupées par le remboursement de leurs dettes. II n'en reste pas moins qu'elles investissent à nouveau à la fois dans la construction et, surtout, dans les autres biens. Intrigués par le recul des investissements observé depuis la fin 2000 (-15\% jusqu'au début 2004), les Instituts se sont penchés sur la question dans leur rapport. Ils parviennent à la conclusion que l'économie
Quelle croissance au $2^{\mathrm{e}}$ semestre?

L'export reste le moteur de l'économie

Une production industrielle bien orientée

Des investissements en accord avec la croissance 
1991-2004: $+1,5 \%$ de croissance moyenne annuelle

Risques :
parité $€ / \$$ et prix du brent

Un frémissement de la consommation?

Taux de chômage : $9,5 \%$ (critères OIT)

Forte progression des petits boulots et emplois aidés allemande se trouve au milieu d'un cycle (d'une durée moyenne de 9 ans 1/2) à faible activité en matière d'investissements, et que la faiblesse des investissements est parfaitement conforme avec la faiblesse de la croissance. Les investissements n'en sont pas moins en progression (+0,9\% au premier trimestre, Destatis).

« Reste la question de savoir pourquoi la croissance est si faible en Allemagne depuis plusieurs années », s'interrogent les Instituts. Elle l'est effectivement, même après la profonde révision statistique entreprise par Destatis dans le cadre de l'harmonisation européenne. Les nouveaux chiffres établis pour la période 1991-2004 donnent certes une croissance légèrement supérieure, mais avec $+1,5 \%$ en moyenne annuelle (au lieu de $+1,3 \%$ ), elle ne modifie en rien la tendance générale : la dynamique de croissance allemande est inférieure à la moyenne européenne depuis plus de dix ans.

L'export en étant toujours pratiquement l'unique moteur, les Instituts s'inquiètent dès lors de savoir comment évolueront les prix des matières premières qui flambent du fait de la vive demande des pays émergents, et ceux des produits pétroliers, soumis actuellement à de fortes fluctuations (leur rapport repose sur I'hypothèse d'un prix moyen de $50 \$$ le baril de brent). Ils s'inquiètent également de l'évolution du taux de change $€ / \$$ (hypothèse : $1 €$ à $1,30 \$$ en moyenne). Si l'euro se déprécie trop, les importations s'en trouveraient renchéries d'autant, l'excédent commercial allemand en pâtirait, se traduisant par une moindre croissance du PIB.

Car la consommation reste toujours aussi faible. Certes, elle avait frémi dans la seconde moitié de 2004, mais au premier trimestre de cette année, elle a de nouveau baissé (-0,2\%, Destatis). Pour les ménages (comme les entreprises, d'ailleurs) le coup de pouce fiscal du début de l'année a été absorbé par la hausse des coûts de l'énergie (voir dans ce numéro). De surcroît, les incertitudes liées à l'emploi et à l'évolution de leurs revenus n'incitent guère les ménages au consumérisme à outrance. Les prix à la consommation ne sont pas en cause, leur hausse est faible. Si les Allemands économisent sur le superflu, ils n'en renouvellent pas moins leur équipement, comme en atteste l'augmentation des immatriculations enregistrée en avril et mai. Autre signe de frémissement: les entrées en commande dans l'industrie des biens de consommation tendent à augmenter depuis quelques mois (+1,8\% en avril/mai par rapport à février/mars).

La situation sur le marché de l'emploi reste tendue, même si la hausse du chômage est largement imputable à l'effet arithmétique des réformes engagées par le gouvernement Schröder. En mai, le nombre de chômeurs recensés par l'Agence fédérale pour l'emploi de Nuremberg était de 4,8 millions, ce qui correspond à un taux de chômage de 11,6 \% selon les critères allemands. Mais selon les critères OIT mis en application par Destatis depuis le début de l'année, le nombre des chômeurs est de 4,06 millions en mai, soit un taux d'inactivité de 9,5\%. La différence entre les deux sources s'explique notamment par le mode de recensement (enquête téléphonique pour l'OIT, données administratives pour Nuremberg) comme par la définition de l'inactivité (disponibilité immédiate vs. inscription à l'Agence). En comparaison internationale (critères OIT), le taux de chômage allemand est donc comparable, voire inférieur au taux français.

Plus intéressante est l'évolution du nombre de chômeurs inscrits si on la met en relation avec le nombre d'actifs, en légère hausse depuis l'an dernier, et avec le nombre des actifs occupés. Ce dernier reflète l'évolution de l'emploi soumis à cotisations sociales, en un mot: l'emploi dit 'normal' ; il stagne. A l'inverse, le nombre des actifs ne cesse d'augmenter, sans effet sur le chômage : cela est dû au fait qu'une partie de la 'réserve occulte' de main d'œuvre (retraités, étudiants, femmes au foyers), celle qui n'est pas enregistrée à l'Agence, a investi massivement les minijobs et autres petits boulots, pourtant initialement créées 
pour les chômeurs de longue durée. Parallèlement, un certain nombre de chômeurs profitent des petits boulots (défiscalisés et non soumis à cotisations, voir REA 67/04) pour 'arrondir leurs fins de mois'. Outre-Rhin, un chômeur peut en effet travailler jusqu'à 15 heures par semaine sans perdre le bénéfice de ses indemnités. Si on y ajoute les 'jobs à $1 €$ ' créées dans le cadre de la loi Hartz IV (voir REA 68/04), le nombre total 'd'emplois' ainsi occupés s'élève à quelque 1,5 million. En mars, quelque 250000 chômeurs avaient en outre monté une Ich-AG, créant ainsi leur propre emploi grâce à des subventions. Cette situation a certes des côtés positifs : elle a permis de réduire le travail au noir et à remettre à disposition du marché de l'emploi une part de la réserve de main d'œuvre. Elle a également entrouvert ce segment à bas salaires qui fait encore défaut à l'économie allemande. Mais elle n'en est pas moins problématique, comme le détaillent les Instituts. Indépendamment des effets d'aubaine qu'elle crée pour les entreprises de certains secteurs (la gastronomie, par exemple), et du fait que ces emplois, non soumis à cotisations sociales et de surcroît largement subventionnés, contribuent à creuser le déficit des régimes de protection sociale (comme du Bund), ceux-ci continuent de maintenir en marge du marché de l'emploi un très grand nombre d'Allemands.

Quant au nombre de chômeurs, il a augmenté de 513000 au premier trimestre (en comparaison de la même période 2004), à la suite de l'entrée en vigueur de la loi Hartz IV, la majeure partie d'entre eux étant d'anciens bénéficiaires de l'aide sociale abrogée par la loi. Cette hausse est donc surtout d'origine administrative et arithmétique (360 000 personnes selon l'Agence de Nuremberg). La situation de l'emploi reste tendue, même si elle est susceptible de s'améliorer légèrement d'ici la fin de l'année. Mais il est difficile, à l'heure actuelle, de faire la part des choses entre les corrections statistiques induites par la mise en place définitive du nouveau dispositif et, surtout, l'évolution de la croissance. II est trop tôt. Et nul ne connaît encore l'effet sur l'emploi et la croissance de la politique que mènera le prochain gouvernement.

Dans ce contexte, et indépendamment des fluctuations que connaissent les différents indicateurs conjoncturels, notamment extérieurs, il faut aussi comprendre les inquiétudes affichées par les divers instituts comme un pessimisme à visée prophylactique... La compétitivité des entreprises allemandes n'est pas en cause ; elle reste solide dans un environnement somme toute porteur. En revanche, la stabilité du futur cap de politique et la consistance des réformes qui seront engagées ou non est actuellement au centre de l'attention générale : les conjoncturistes font pression sur le monde politique pour lui rappeler l'impérieuse nécessité de la poursuite des réformes structurelles. Dès avant même le revers électoral de la coalition SPD/Verts en Rhénanie du Nord-Westphalie, l'ardeur réformiste du gouvernement fédéral SPD/Verts commençait à faiblir, ce qui avait amené les Instituts à rappeler quelques priorités au gouvernement Schröder dans leur rapport d'avril. Elles valent pareillement pour son successeur.

La priorité absolue est celle-ci : « des budgets publics consolidés sont la condition essentielle pour le succès des réformes ». Au nom du développement durable ou de la solidarité générationnelle, le Pacte de stabilité doit impérativement être respecté, même si sa révision «a détérioré les conditions » d'une réelle consolidation. II est vrai que la loi budgétaire n'en prend pas le chemin. Le quotidien Frankfurter Allgemeine Zeitung (05-06-05) se complaît à rappeler le montant cumulé de la dette allemande : « $1436983500554 €$ » très exactement. Le seul service de la dette du Bund (881 milliards € ; le restant étant la dette des autres collectivités) ponctionne cette année son budget de 26,9 milliards $€$ - à mettre en regard de 187 milliards $€$ de recettes. La moitié de ses dépenses (47\%) est réservée au financement des retraites et du coût du chômage ; les mesures Hartz accaparent plus de 40 milliards $€$, soit la moitié du budget réservé aux retraites. En comparaison, les investissements s'élèvent à un peu moins de 23 milliards $€$ seulement.
Incertitudes quant à la politique du prochain gouvernement

Dette allemande : $1436983500554 €$ 
Les économistes lancent un appel pressant aux réformes
Le 30 juin, pour la première fois dans l'histoire allemande (un seul précédent est connu: le débat sur l'introduction de la monnaie unique dans les années 1990), 241 économistes allemands de toute obédience, dont les plus réputés, ont publié un appel aux réformes : « l'Appel de Hambourg ». Regrettant « un manque effarant de compétences économiques » dans le concert des arguments électoralistes de tous, ils poursuivent: "Nous en appelons au sens des responsabilités des élus du peuple afin qu'ils résistent à la tentation des solutions simplistes et que, au contraire, ils apportent des réponses non édulcorées aux brûlantes préoccupations économiques ». II convient d'informer les citoyens sur la réalité de la crise structurelle et des réformes indéniablement douloureuses qu'elle requiert. A cet effet, les économistes proposent au prochain gouvernement un programme en 11 points dont on peut résumer ainsi les orientations : réduire drastiquement les dépenses de l'Etat ; déréguler les marchés ; baisser résolument les planchers salariaux ; investir, notamment dans les technologies d'avenir ; impulser une logique de performance et de compétitivité au système de formation; en revenir à une saine appréciation de la concurrence ; et saisir la globalisation des activités comme une chance pour le site Allemagne. Reste à savoir le sort que le prochain gouvernement réservera à ce régime draconien. Sa priorité en tout cas devra être de donner confiance aux acteurs économiques - consommateurs et entreprises pareillement.

Isabelle Bourgeois (13-07-2005)

Prévisions du rapport de printemps 2005 des instituts de conjoncture

\begin{tabular}{|c|c|c|c|c|c|c|}
\hline & 2003 & 2004 & 2005 & Sem. 1 & Sem. 2 & 2006 \\
\hline \multicolumn{7}{|c|}{ (variation en \% par rapport à la même période de l'année précédente) } \\
\hline $\begin{array}{l}\text { PIB } \\
\text { Est } \\
\text { Ouest (Berlin inclus) }\end{array}$ & $\begin{array}{r}-0,1 \\
0,2 \\
-0,2\end{array}$ & $\begin{array}{l}1,6 \\
1,5 \\
1,6\end{array}$ & $\begin{array}{l}0,7 \\
0,7 \\
0,7\end{array}$ & $\begin{array}{l}- \\
- \\
-\end{array}$ & $\begin{array}{l}- \\
- \\
-\end{array}$ & $\begin{array}{l}1,5 \\
1,5 \\
1,5\end{array}$ \\
\hline $\begin{array}{l}\text { Consommation privée } \\
\text { Consommation publique }\end{array}$ & $\begin{array}{l}0,0 \\
0,1\end{array}$ & $\begin{array}{r}-0,4 \\
0,4\end{array}$ & $\begin{array}{r}0,4 \\
-0,1\end{array}$ & - & - & $\begin{array}{l}0,7 \\
0,4\end{array}$ \\
\hline $\begin{array}{l}\text { Investisssements bruts en biens d'équipt. } \\
\text { Equipement et autres biens } \\
\text { Construction }\end{array}$ & $\begin{array}{l}-2,2 \\
-0,9 \\
-3,2\end{array}$ & $\begin{array}{r}-0,9 \\
1,2 \\
-2,8\end{array}$ & $\begin{array}{r}0,7 \\
3,8 \\
-2,2\end{array}$ & $\begin{array}{l}- \\
-\end{array}$ & $\begin{array}{l}- \\
-\end{array}$ & $\begin{array}{r}1,5 \\
4,5 \\
-1,4\end{array}$ \\
\hline $\begin{array}{l}\text { Demande intérieure } \\
\text { Exportations } \\
\text { Importations }\end{array}$ & $\begin{array}{l}0,5 \\
1,8 \\
4,0\end{array}$ & $\begin{array}{l}0,4 \\
8,6 \\
6,4\end{array}$ & $\begin{array}{l}0,4 \\
4,1 \\
3,8\end{array}$ & $\begin{array}{l}- \\
-\end{array}$ & $\begin{array}{l}- \\
-\end{array}$ & $\begin{array}{l}0,9 \\
6,4 \\
5,7\end{array}$ \\
\hline $\begin{array}{l}\text { Revenu national brut } \\
\text { Rev. brut issu du travail salarié } \\
\text { Rev. brut issu des entr. et de la propté. }\end{array}$ & $\begin{array}{l}- \\
-\end{array}$ & $\begin{array}{r}2,7 \\
0,1 \\
10,4\end{array}$ & $\begin{array}{l}1,5 \\
0,4 \\
4,1\end{array}$ & $\begin{array}{l}2,7 \\
1,2 \\
7,0\end{array}$ & $\begin{array}{l}2,7 \\
1,5 \\
6,2\end{array}$ & $\begin{array}{l}2,7 \\
1,3 \\
6,6\end{array}$ \\
\hline Revenu disponible & - & 1,2 & 2,1 & 2,4 & 2,0 & 2,2 \\
\hline Consommation des ménages & - & 1,2 & 2,1 & 2,5 & 2,0 & 2,2 \\
\hline Epargne des ménages & - & 2,7 & 2,2 & 2,5 & 2,2 & 2,3 \\
\hline Prix à la consommation b) & 1,1 & 1,6 & 1,7 & - & - & 1,5 \\
\hline Coûts salariaux unitaires & 0,7 & $-1,1$ & $-0,0$ & - & - & 0,0 \\
\hline \multicolumn{7}{|c|}{ (état) } \\
\hline $\begin{array}{l}\text { Déficit public en } \% \text { du PIB } \\
\text { Dette publique (en milliards } € \text { ) }\end{array}$ & $\begin{array}{r}-3,0 \\
-81,3\end{array}$ & $\begin{array}{r}-3,7 \\
-80,3\end{array}$ & $\begin{array}{l}-3,4 \\
-76,1\end{array}$ & - & - & $\begin{array}{r}-3,3 \\
-74,0\end{array}$ \\
\hline $\begin{array}{l}\text { Actifs (en millions) } \\
\text { Est, Berlin inclus (en millions) } \\
\text { Ouest (en millions) }\end{array}$ & $\begin{array}{r}38,31 \\
7,52 \\
30,74\end{array}$ & $\begin{array}{r}38,44 \\
7,54 \\
30,85\end{array}$ & $\begin{array}{c}38,73 \\
7,60 \\
31,08\end{array}$ & $\begin{array}{l}- \\
-\end{array}$ & $\begin{array}{l}- \\
-\end{array}$ & $\begin{array}{r}39,07 \\
7,67 \\
31,35\end{array}$ \\
\hline $\begin{array}{l}\text { Chômeurs (en millions) }{ }^{\text {d) }} \\
\text { Est, Berlin inclus (en millions) } \\
\text { Ouest (en millions) }\end{array}$ & $\begin{array}{l}4,38 \\
1,62 \\
2,75\end{array}$ & $\begin{array}{l}4,38 \\
1,60 \\
2,78\end{array}$ & $\begin{array}{l}4,84 \\
1,67 \\
3,17\end{array}$ & $\begin{array}{l}- \\
-\end{array}$ & $\begin{array}{l}- \\
-\end{array}$ & $\begin{array}{l}4,52 \\
1,56 \\
2,96\end{array}$ \\
\hline $\begin{array}{l}\text { Taux de chômage (en \%) } \\
\text { Est, Berlin inclus } \\
\text { Ouest }\end{array}$ & $\begin{array}{r}10,3 \\
17,8 \\
8,2\end{array}$ & $\begin{array}{r}10,2 \\
17,5 \\
8,3\end{array}$ & $\begin{array}{c}11,1 \\
18,0 \\
9,3\end{array}$ & $\begin{array}{l}- \\
- \\
-\end{array}$ & $\begin{array}{l}- \\
-\end{array}$ & $\begin{array}{r}10,4 \\
16,9 \\
8,6\end{array}$ \\
\hline Taux d'épargne & - & 10,9 & 10,9 & 12,4 & 9,4 & 10,9 \\
\hline
\end{tabular}

Source des données : Rapport de printemps des six instituts de conjoncture DIW (Berlin), HWWA (Hambourg), ifo (Munich), IfW (Kiel), IWH (Halle), RWI (Essen), en date du 26-04-2005. Données établies selon le Système européen des comptes nationaux (SEC 95); 2005 et 2006 ; prévisions des Instituts. a) ménages et organismes privés à but non lucratif ; b) $2000=$ indice 100 ; c) données Destatis; d) données administratives de la BA de Nuremberg (les données Destatis ou d'Eurostat, établies selon les critères de l'OIT, sont inférieures d'un point de pourcentage environ) ; à partir de 2004 : hors mesures de requalification. 